Research Paper

\title{
Can muscle vibration be the future in the treatment of cerebral palsy-related drooling? A feasibility study.
}

\author{
Emanuele F. Russo ${ }^{1}$, Rocco S. Calabrò ${ }^{\circledR}{ }^{\bowtie}$, Patrizio Sale ${ }^{3}$, Filomena Vergura ${ }^{1}$, Maria C. De Cola, ${ }^{1}$ Angela \\ Militi, ${ }^{4}$ Placido Bramanti, ${ }^{2}$ Simona Portaro, ${ }^{2}$ and Serena Filoni ${ }^{1}$ \\ 1. Padre Pio Foundation and Rehabilitation Centers, San Giovanni Rotondo, Foggia, Italy; \\ 2. IRCCS Centro Neurolesi Bonino-Pulejo, Messina, Italy; cristina.decola@gmail.com \\ 3. Rehabilitation Unit, Department of Neurosciences, University of Padua; \\ 4. Dipartimento di Scienze Biomediche odontoiatriche e delle immagini Morfologiche e Funzionali, University of Messina, Italy \\ $\square$ Corresponding author: Rocco S. Calabrò, IRCCS Centro Neurolesi Bonino-Pulejo, Via Palermo, Cda Casazza, SS113, 98124 Messina, Italy. Phone/Fax \\ +3909060128166; salbro77@tiscali.it
}

(1) The author(s). This is an open access article distributed under the terms of the Creative Commons Attribution License (https://creativecommons.org/licenses/by/4.0/). See http://ivyspring.com/terms for full terms and conditions.

Received: 2019.03.13; Accepted: 2019.07.05; Published: 2019.09.20

\begin{abstract}
Background: Drooling is an involuntary loss of saliva from the mouth, and it is a common problem for children with cerebral palsy (CP). The treatment may be pharmacological, surgical, or speech-related. Repeated Muscle Vibration (rMV) is a proprioceptive impulse that activates fibers la reaching the somatosensory and motor cortex. Aim: The aim of the study is to evaluate the effectiveness of $r M V$ in the treatment of drooling in CP. Design, setting and population: This was a rater blinded prospective feasibility study, performed at the "Gli Angeli di Padre Pio" Foundation, Rehabilitation Centers (Foggia, Italy), involving twenty-two CP patients affected by drooling (aged 5-15, mean 9,28 $\pm 3,62$ ). Children were evaluated at baseline (T0), 10 days (T1), 1 month (T2) and 3 months (T3) after the treatment. Methods: The degree and impact of drooling was assessed by using the Drooling Impact Scale (DIS), the Drooling Frequency and Severity Scale (DFSS), Visual Analogue Scale (VAS) and Drooling Quotient (DQ). An rMV stimulus under the chin symphysis was applied with a $30 \mathrm{~min}$ protocol for 3 consecutive days. Results: The statistical analysis shows that DIS, DFSS, VAS, DQ improved with significant differences in the multiple comparisons between $\mathrm{Tl}$ vs $\mathrm{T} 2, \mathrm{Tl}$ vs $\mathrm{T} 3$ and $\mathrm{Tl}$ vs $\mathrm{T} 4$ ( $\mathrm{p} \leq 0.001)$. Conclusion This study demonstrates that $\mathrm{rMV}$ might be a safe and effective tool in reducing drooling in patients with CP. The vibrations can improve the swallowing mechanisms and favor the acquisition of the maturity of the oral motor control in children with CP.
\end{abstract}

Key words: Muscle vibration; neurorehabilitation; developmental disorders; sialorrhea.

\section{Introduction}

The widespread incidence of Cerebral palsy (CP) in childhood is 1-5 per 1000 live births [1], and it is the most frequent motor disability during this period. CP is considered a neurological disorder caused by a non-progressive brain injury or malformation that occurs while the child's brain is under development. The disease primarily affects body movement and muscle coordination, but may determine intellectual disabilities and behavioral abnormalities. Sometimes there could be epilepsy and secondary musculoskeletal problems [2].
Saliva has a fundamental role in keeping humid the mouth and preserving oral hygiene, making the bolus smooth while swallowing and regulating esophageal acidity. The submandibular glands (70\%) produce the majority of saliva and only $30 \%$ is produced by the other glands [3].

The incapacity of controlling saliva in the mouth is due to poor head and lip control and/or tongue incoordination with a mouth constantly open or an diminished tactile sensation. Other causes can be macroglossia, nasal obstruction or dental 
malocclusion [4].

The incapacity to tackle oral secretions caused by oro-motor disorders is termed drooling or sialorrhea. Until the age of 18 months, drooling is normal, and it is accepted until the age of four. [5-7]. Drooling may affect up to $45 \%$ of CP patients, and can be classified into anterior and posterior. The former is clinically visible and it occurs in the oral phase of swallowing, whilst the latter is concerned with the spilling of saliva on the tongue due to the facial isthmus, and it regards the pharyngeal phase in patients with serious oropharyngeal dysphagia [5-8].

Drooling can cause distress and affliction not only in children, but also in parents and caregivers, due to bad smelling, irritated or macerated facial skin, orofacial infections, dehydration, speech and masticatory problems [9]. The probability of aspiration pneumonia and chest infections are higher. Unfortunately, all these problems can lead to social isolation. [9]. The various available treatments include anticholinergic drugs, rehabilitation, kinesio-taping, botulinum toxin injection and, in specific cases, surgery [10-13].

Some studies have assessed the validity of rehabilitation by using oro-motor therapy, behavioral approaches and biofeedback. The use of sublingual, oral and cutaneous medication (muscarinic cholinergic receptor antagonists) is now limited because there is very little evidence of its validity and it may have side effects [14]. Although some studies have demonstrated the efficacy of botulinum toxin [12], the best approach to this devastating problem has not been determined yet [15-16].

The use of vibratory stimuli has demonstrated practical applications in the areas of therapeutic rehabilitation and exercise performance. Muscle vibration is a technique that applies a low-amplitude/high-frequency vibratory stimulus to a specific muscle using a mechanical device. Repeated Muscle Vibration (rMV) is a proprioceptive impulse that activate fibers Ia reaching the somatosensory and motor cortex. rMV has been employed in rehabilitation in many cases with considerable results. It has been demonstrated that rMV may reduce spasticity [17], and facilitate motor control tasks [18], improve fatigue resistance, time of force development and strength [19], intensify muscle contraction [20], and improve gait [21].

The aim of the study is to evaluate a new technique based on rMV for the treatment of drooling in patients with $\mathrm{CP}$. We postulated that $\mathrm{rMV}$ might improve drooling by boosting oral motor control, considering its positive effects on muscle coordination and strength.

\section{Materials and methods}

\section{Study design and population}

This was a rater blinded prospective pilot study performed at the "Gli Angeli di Padre Pio" Foundation, Rehabilitation Centers, Foggia, Italy. Among the $50 \mathrm{CP}$ patients screened for study enrolment, twenty-two children met the inclusion criteria and entered the study. The children $(8$ males, 14 females, aged 5 - 15 years, mean 9,28 $\pm 3,62$ ) were enrolled from February 2016 to April 2018.

Inclusion criteria were: i) confirmed diagnosis of cerebral palsy, ii) score of $\geq 6$ on DFSS, iii) age between 5 and 18 years, and iv) informed consent obtained by the parents/caregivers. Exclusion criteria were: i) previous surgical interventions for saliva control, ii) use of drugs that could interfere with saliva secretion (including botulinum toxin) and iii) involvement in other medical studies.

This study was approved by the Local ethics committee (IRCCSME-ID 29/2015), and was performed in accordance with the Declaration of Helsinki. All parents or caregivers gave their informed consent for this study.

Muscle vibration was applied by means of the Cro®System (Pacioni\&C. S.n.c, Italy), an electromechanical transducer with a particular mechanical support. We used a low amplitude rMV at a fixed frequency of $100 \mathrm{~Hz}$. Thanks to a little probe (diameter of $10 \mathrm{~mm}$ ), the vibration was located over submandibular muscles, behind mandibular symphysis, i.e. digastric, mylohoyid, hyoglossus, geniohyoid, genioglossus and styloglossus muscles (Fig. 1). The transducer was directed so that it produced sinusoidally modulated forces ranging between 7 and $9 \mathrm{~N}$. The range of vibration amplitude was from 0.05 to $0.1 \mathrm{~mm}$.

The training lasted 3 consecutive days, and was performed three times a day. Every application lasted 10 minutes and there was an interval of 60 s between the three applications, so that the person's muscles could relax.

\section{Outcome Measures}

Children were evaluated by a skilled speech therapist, at baseline (T0), 10 days (T1), 1 month (T2) and 3 months (T3) after the treatment.The degree and impact of drooling was assessed by means of the Drooling Impact Scale (DIS) and with the Drooling Frequency and Severity Scale (DFSS), Visual Analogue Scale (VAS), Drooling Quotient (DQ). Every measurement was performed in the morning under normal conditions about 1 hour after mealtime.

The DQ (expressed as a percentage) is a method, which is semi-quantitative obtained by observation. 
After having wiped off the saliva from the chin and any trace of food that had remained in the mouth was taken away too, the drooling quotient assessment started. DQ was recorded, registering the episodes of drooling that took place during two stages of 5 minutes that were separated by an interval of 30 minutes [22]. When new saliva appeared on the lip margin or drooling started from the chin, it was considered as an episode of drooling. Every 15 seconds, for 5 minutes (totally 20 assessments) there was a control to verify if drooling was occurring or not. During the DQ 'rest' condition, the child could watch TV, sit in an upright position on his wheelchair, but he did not have to talk. In the DQ "activity" condition, according to the child's interests and his abilities they could perform different activities such as using electronic communication devices or play building blocks.

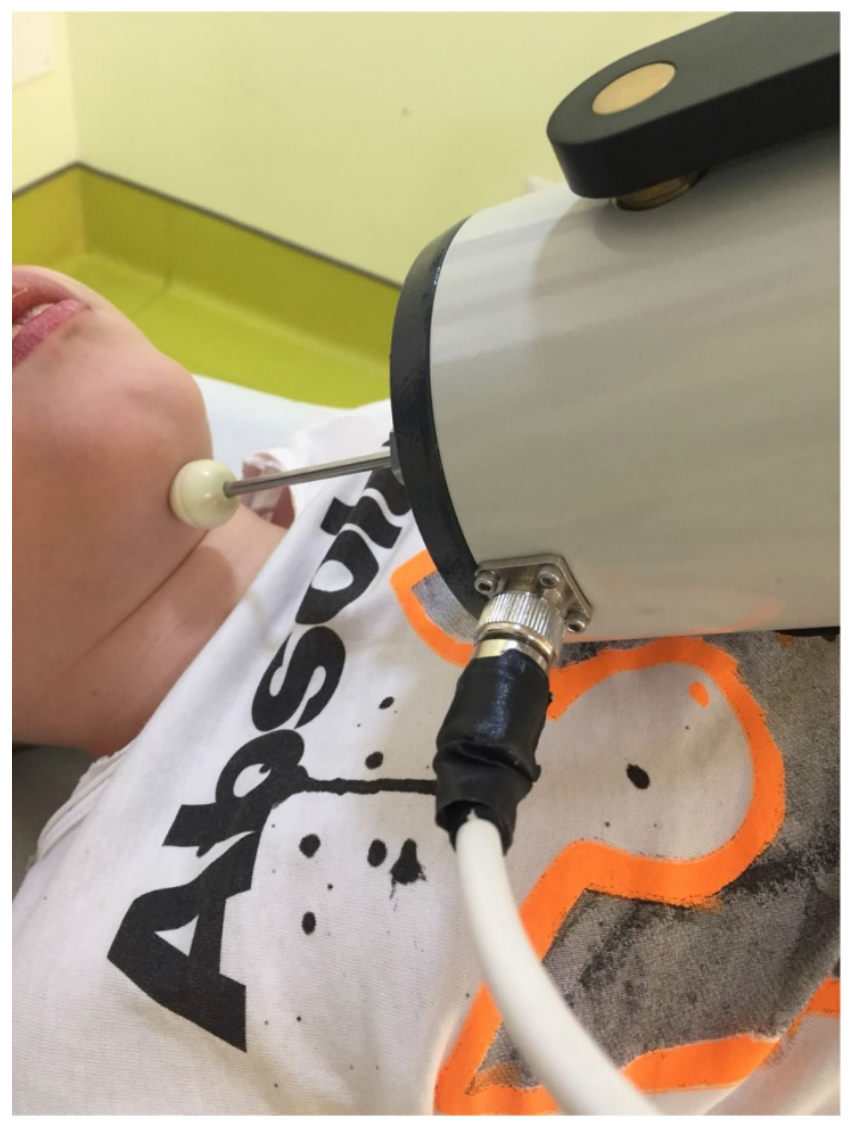

Fig. 1 shows the transducer position during drooling treatment in a patient with cerebral palsy.

In the DIS questionnaire that we distributed the week before, there were 10 questions rated from 1 to 10 on a semantic differential scale [23]. The total scoring of the questionnaire gives a general evaluation on the impact that drooling has on the child and the severity of drooling. The maximum possible total for the scale was 100 . To evaluate the frequency of drooling and its severity the DFSS scale was adopted [24]. Every person was attributed with a grade that corresponded to these definitions: 1, dry (when there was no drooling); 2, mild (when only the lips were wet); 3 , moderate (when the lips and chin were wet); 4 , severe (when drooling wetted clothes); 5 , profuse (when it wetted clothes, hands and objects). The frequency of drooling was rated too: 1, no drooling; 2, sporadic drooling; 3, repeated drooling, 4, unceasing drooling. Taking into consideration the values of both scales, a combined drooling scale was formed that went from 2 to 9 . In addition, the parents were administered a VAS scale, to get their impression on the symptom severity ( 0 absence of drooling, $100=$ exaggerated drooling).

\section{Statistical analysis}

The normality of the distribution of all variables was assessed by the Shapiro -Wilk statistic. Data are reported as Median and Interquartile Range (IQR). For every outcome variable taken into consideration, to prove the differences among the different assessment period, the Friedman test was adopted, after that Wilcoxon signed rank test and Holm-Bonferroni sequential correction were carried out for multiple comparisons [25].

For every analysis $\mathrm{p}$ values $<0.05$ were regarded significant in terms of statistics.

\section{Results}

All participants completed the three sessions of the treatment, without reporting any significant adverse event. Table 1 summarizes the participants' characteristics.

Friedman test results were significant for all clinical test scores administered, demonstrating a significant reduction among the assessment time points in DQREST $(x 2(3)=29.099, p \leq 0.001)$, DQACT $(x 2(3)=35.250, p \leq 0.001)$, DIS $(x 2(3)=34.422$, $p \leq 0.001)$, VAS $(x 2(3)=31.010, p \leq 0.001)$ and DFSS $\left(x^{2}\right.$ $(3)=34.153, p \leq 0.001)$ scores. Indeed, we found a significant reduction in frequency, intensity and severity of drooling at rest and during patient's activities (Fig 2).

However, the post-hoc analysis revealed statistically significant differences only between baseline (T0) and the other assessment time points (T1, T2 and T3), as showed in Table 2. Hence, the score changes are significant from baseline to post-treatment and after the improvement remains stable (table 3). 

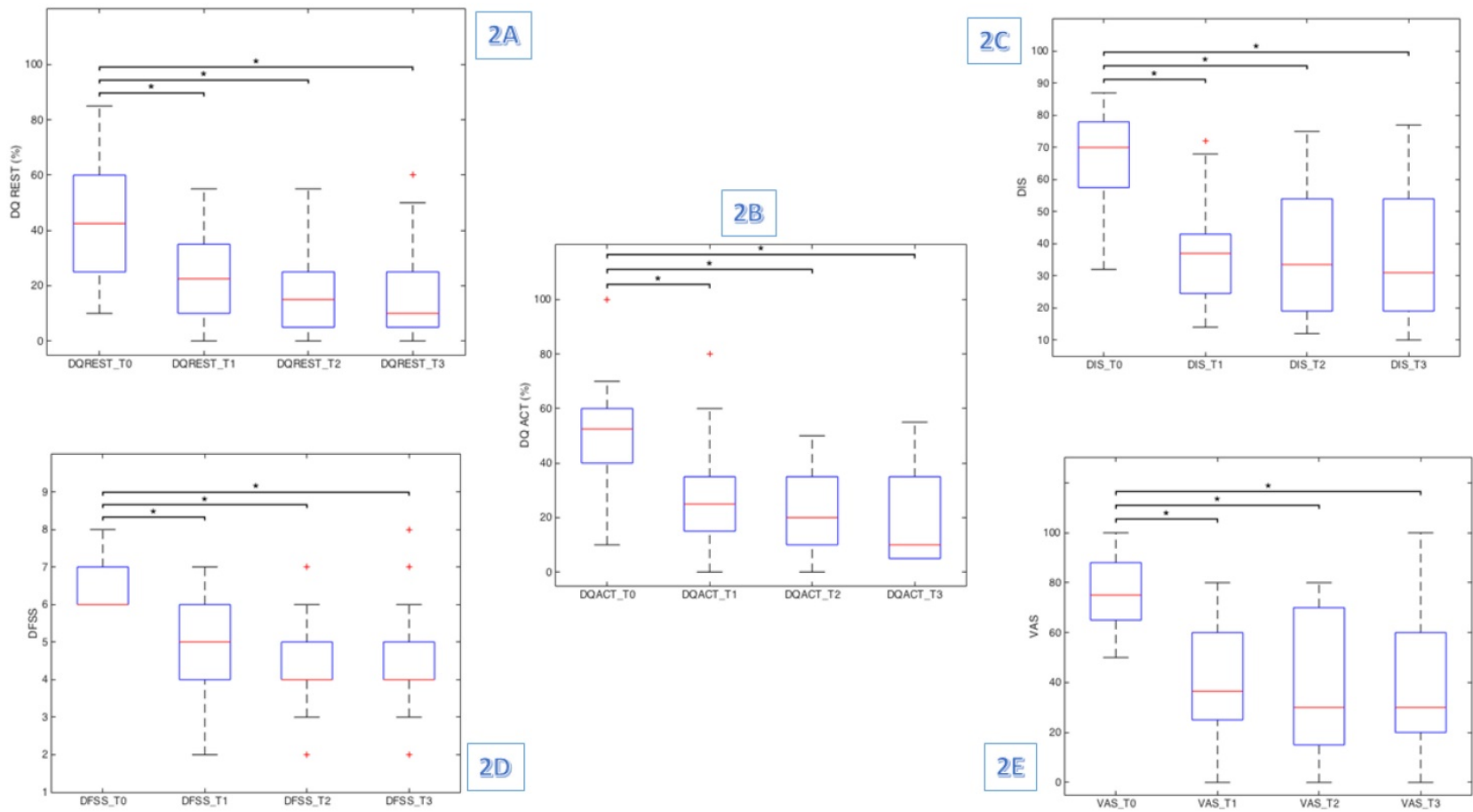

Fig. 2 shows the box plot diagram for a) DQREST, b) DQACT, c) DIS, d) DFSS and e) VAS. * Wilcoxon signed rank test (Holm- Bonferroni sequential correction)

Table 1. Characteristics of the sample.

\begin{tabular}{ll}
\hline Participants & 22 \\
\hline Age (years) & $9,68 \pm 3,45$ \\
Affected side & 1 \\
Unilateral & 21 \\
Bilateral & \\
Cerebral palsy subtype & 20 \\
Spastic & 2 \\
Dyskinetic & 0 \\
Ataxic & \\
Comorbid Factors & 8 \\
Epilepsy & 20 \\
Intellectual disability & \\
Food intake & 4 \\
Unimpaired & 17 \\
Dysphagic & 1 \\
Gastronomy tube & \\
Speech & 1 \\
Unimpaired & 12 \\
Dysarthric & 9 \\
Anarthric & The quantitative variable age is expressed as mean \pm standard deviation, whereas \\
the qualitative variables as absolute frequencies.
\end{tabular}

\section{Discussion}

To the best of our knowledge, this is the first attempt to evaluate the effect of focal muscle vibration in the treatment of sialorrhea. Our pilot study support our idea that rMV could be a valuable tool to improve drooling in children with CP. Indeed, we found a significant reduction in frequency, intensity and severity of drooling at rest and during patient's activities, as demonstrated by the clinical test administered before and after the treatment.
Table 2. Median and IQR of evaluation at baseline (T0), post-treatment (T1) and two follow-up (T2-T3).

\begin{tabular}{|c|c|c|c|c|}
\hline & $\begin{array}{l}\text { Baseline (T0) } \\
\text { Median (IQR) }\end{array}$ & $\begin{array}{l}\text { Post-treatment } \\
\text { (T1) } \\
\text { Median (IQR) }\end{array}$ & $\begin{array}{l}\text { Follow-up (T2) } \\
\text { Median (IQR) }\end{array}$ & $\begin{array}{l}\text { Follow-up } \\
\text { (T3) } \\
\text { Median (IQR) }\end{array}$ \\
\hline DFSS & $6.0(6.0-7.0)$ & $5.0(4.0-6.0)$ & $4.0(4.0-5.0)$ & $4.0(4.0-5.0)$ \\
\hline DIS & $70.0(57.1-78.0)$ & $37.0(23.9-47.2)$ & $33.5(18.5-55.0)$ & $\begin{array}{l}31.0 \\
(18.8-54.5)\end{array}$ \\
\hline DQACT (\%) & $\begin{array}{l}52.5(40.0- \\
61.2)\end{array}$ & $25.0(15.0-36.2)$ & $20.0(10.0-35.0)$ & $10.0(5.0-35.0)$ \\
\hline $\begin{array}{l}\text { DQREST } \\
(\%)\end{array}$ & $42.5(23.7-62.5)$ & $22.5(8.7-36.2)$ & $15.0(5.0-26.2)$ & $10.0(3.7-26.2)$ \\
\hline VAS & $75.0(63.7-88.5)$ & $36.5(25.0-60.0)$ & 30.0 (13.7-70.0) & $\begin{array}{l}30.0 \\
(18.7-62.5)\end{array}$ \\
\hline
\end{tabular}

Table 3. Friedman's test and Wilcoxon signed rank test (with Holm- Bonferroni sequential correction) results.

\begin{tabular}{|c|c|c|c|c|c|c|c|}
\hline & \multirow{2}{*}{$\begin{array}{l}\text { Friedman } \\
\text { test } \\
\text { p-value }\end{array}$} & \multicolumn{6}{|c|}{ Wilcoxon signed-rank test } \\
\hline & & $\begin{array}{l}\text { p-value } \\
\text { T0-T1 }\end{array}$ & $\begin{array}{l}\mathrm{p} \text {-value } \\
\text { T0-T2 }\end{array}$ & $\begin{array}{l}\text { p-value } \\
\text { T0-T3 }\end{array}$ & $\begin{array}{l}\text { p-value } \\
\text { T1-T2 }\end{array}$ & $\begin{array}{l}\text { p-value } \\
\text { T1-T3 }\end{array}$ & $\begin{array}{l}\text { p-value } \\
\text { T2-T3 }\end{array}$ \\
\hline DFSS & $<0.001$ & $<0.001$ & $<0.001$ & $<0.001$ & 0.090 & 0.112 & 0.999 \\
\hline DIS & $<0.001$ & $<0.001$ & $<0.001$ & $<0.001$ & 0.666 & 0.808 & 0.808 \\
\hline DQACT $(\%)$ & $<0.001$ & $<0.001$ & $<0.001$ & $<0.001$ & 0.234 & 0.168 & 0.234 \\
\hline $\begin{array}{l}\text { DQREST } \\
(\%)\end{array}$ & $<0.001$ & $<0.001$ & $<0.001$ & $<0.001$ & 0.204 & 0.340 & 0.647 \\
\hline VAS & $<0.001$ & $<0.001$ & $<0.001$ & $<0.001$ & 0.999 & 0.999 & 0.999 \\
\hline
\end{tabular}

Besides the medical problems, this annoying symptom can be considered a social disability, as it becomes an obstacle for social interaction. Consequently, it has a negative impact on both $\mathrm{CP}$ 
patients and caregivers' quality of life, being drooling quite common in such neurological disorder. Although drooling may have different causes, in $\mathrm{CP}$ patients it is more due to a disturbed deglutition than to hypersalivation [26]. Indeed, due to neurodevelopmental delay there could be a disturbance of some primary functions, including oral sensibility, swallowing, lip closure, and suction. However, in the presence of saliva overflow, the most probable cause is an incoordination of tongue mobility, as it has been demonstrated that the quantity of saliva produced remains constant. In CP patients an abnormal coordination of head and trunk, and orofacial and palatolingual musculature, should be also considered [27-29].

Thus, although many factors may contribute to drooling, the problem in CP depends mainly on the lack of oral motor control. Motor control and muscle strengthening can be influenced by a powerful proprioceptive stimulation, as rMV reaches undeviatingly both the SI and MI by activating (at low amplitudes) Ia afferent fibers. The straight connections between SI and MI cortices supplies the anatomical substrate necessary for the function played by $\mathrm{MV}$ in reorganizing the motor and somatosensory cortices [17].

This is the reason why, for the ever first time, we decided to use rMV for the treatment of drooling in these patients.

Although there are different therapeutic ways of treating sialorrhea, we believe that rMV could be of some help in improving the disabling symptom. Speech therapy training could be a good solution because it treats the causes and its long-term effects, but it depends entirely on the child's intellectual capacities and, besides, the treatment has to be repeated with regular frequency too [4]. The rMV treatment, instead, has the advantage of being applied in shorter sessions and its validity depends only on the correct target-muscle positioning of the transducer.

Botulinum toxin is valid and safe treatment of sialorrhea, but it has many disadvantages $[11,15,16]$. Indeed, it is only temporarily effective, as every 3-6 months the patient should repeat it, and it is very expensive due to both the drug costs and the need of highly qualified multidisciplinary staff $[4,11]$. Moreover, the main disadvantage of the treatment is that it concentrates only on the effect (i.e. reduction of saliva production) and not on the causes (i.e. oral motor incoordination), as instead rMV does [12, 26].

The surgical treatment, although definitive and valid, should be considered only in selected cases, as it needs a specialized team and general anesthesia [10]. Finally, the use of specific drugs, such as anticholinergics, may lead to undesirable and harmful side effects, including insomnia, irritability, diarrhea and vomiting [14].

Differently from most of the previous studies, the rMV approach acts on the cause of drooling and not on the effects. rMV can also be used in non-collaborating individuals (as most of the patients with intellectual disability are) that would not allow them to actively participate in a speech therapy training, as those enrolled in our study.

Notably, we found that the improvement of drooling was already evident one week after the end of the treatment and lasted up to three months. The treatment was well tolerated and safe, there were no adverse events and dropouts.

We have applied focal vibrations under the chin symphysis to stimulate the muscles behind mandibular symphysis, i.e. digastric, mylohoyid, hyoglossus, geniohyoid, genioglossus and styloglossus muscles. This vibratory stimulus may therefore have affected the orofacial and lingual palate mechanisms, improving coordination, muscle tone, strengthening the muscles and giving a great sensorial stimulus.

Indeed, it is possible that the improvement of the coordination and strength mechanisms of the treated muscles could favor the improvement of swallowing through the acquisition of a maturity of the oral motor control. Consequently, the best management of saliva inside the mouth causes an immediate improvement of the drooling. Swallowing, once acquired, is constantly trained in the activities of daily life, thus probably potentiating the effects of the treatment, also at follow-up.

The rMV training could have boosted connectivity within the sensorimotor areas by activating Ia fibers [31, 32]. Indeed, such higher vibration frequencies have been shown to elicitate motor response, spinal and supra spinal reflexes and the activation of suprasegmental structures so to modify motor command strategies. More in detail, it has been shown that the rMV-induced modifications are very likely due to at least 2 forms of plasticity: i) a form of nonsynaptic plasticity that induces changes in the intrinsic properties of neural membranes (explaining the lowering of the motor threshold), and ii) a Hebbian-like mechanism of synaptic plasticity, which may account for the functional restoration of inactivated, though preserved, motor pathways and/or rearrangements of motor cortical maps [17].

This is the reason why we may argue that the use of vibrations is an effective and potentially long-lasting method for treatment for sialorrhea.

The main limitations of this study include the low number of participants and the absence of a 
control group. However, this is a pilot study, and further larger sample randomized trials are needed to confirm these findings and investigate the factors related to non-responder $\mathrm{CP}$ patients. Moreover, patients were followed for a short period, thus studies with long term follow-up should be encouraged to evaluate the persistence of rMV after effects.

\section{Conclusions}

This study demonstrates that the treatment of drooling with rMV in children with $\mathrm{CP}$ is safe, well tolerated, and effective up to three months after the end of treatment. According to our findings, focal vibrations may improve swallowing mechanisms and favor the acquisition of the maturity of the oral motor control. Moreover, the reduction of the drooling improves the quality of life of the little patients and their caregivers.

\section{Competing Interests}

The authors have declared that no competing interest exists.

\section{References}

1. Lee RW, Poretti A, Cohen JS, et al. A diagnostic approach for cerebral palsy in the genomic era. Neuromolecular Med. 2014;16(4):821-844.

2. Rosenbaum $\mathrm{P}$, Paneth $\mathrm{N}$, Leviton $\mathrm{A}$, et al. A report: the definition and classification of cerebral palsy April 2006. Dev Med Child Neurol Suppl. 2007;109:8-14.

3. Bouchoucha M, Callais F, Renard P, Ekindjian OG, Cugnenc PH, Barbier JP. Relationship between acid neutralization capacity of saliva and gastro-oesophageal reflux. Arch Physiol Biochem. 1997;105(1):19-26.

4. Bavikatte G, Sit PL, Hassoon A. Management of drooling of saliva. Br J Med Pract. 2012;5(1)

5. Blasco P. Prevalence and predictors of drooling. Dev Med Child Neurol. 2012;54(11):970.

6. Chang S-C, Lin C-K, Tung L-C, Chang N-Y. The association of drooling and health-related quality of life in children with cerebral palsy. Neuropsychiatr Dis Treat. 2012;8:599-604

7. Tahmassebi JF, Curzon MEJ. Prevalence of drooling in children with cerebral palsy attending special schools. Dev Med Child Neurol. 2003;45(9):613-617.

8. Weir K, McMahon S, Barry L, Ware R, Masters IB, Chang AB. Oropharyngeal aspiration and pneumonia in children. Pediatr Pulmonol. 2007;42(11):1024-1031.

9. van der Burg JJW, Jongerius PH, van Limbeek J, van Hulst K, Rotteveel JJ. Social interaction and self-esteem of children with cerebral palsy after treatment for severe drooling. Eur J Pediatr. 2006;165(1):37-41.

10. Blasco P. The treatment of drooling. Dev Med Child Neurol. 2010;52(11):984

11. Reddihough D, Erasmus CE, Johnson H, McKellar GMW, Jongerius PH. Botulinum toxin assessment, intervention and aftercare for paediatric and adult drooling: international consensus statement. Eur J Neurol. 2010;17 Suppl 2:109-121.

12. Walshe $M$, Smith $M$, Pennington $L$. Interventions for drooling in children with cerebral palsy. Cochrane Database Syst Rev. 2012;(2):CD008624

13. Mikami DLY, Furia CLB, Welker AF. Addition of Kinesio Taping of the orbicularis oris muscles to speech therapy rapidly improves drooling in children with neurological disorders. Dev Neurorehabil. September 2017:1-6.

14. Jongerius $\mathrm{PH}$, van Tiel P, van Limbeek J, Gabreels FJM, Rotteveel JJ. A systematic review for evidence of efficacy of anticholinergic drugs to treat drooling. Arch Dis Child. 2003;88(10):911-914.

15. Porte $\mathrm{M}$, Chaleat-Valayer $\mathrm{E}$, Patte $\mathrm{K}$, $\mathrm{D}^{\prime}$ Anjou $\mathrm{M}-\mathrm{C}$, Boulay $\mathrm{C}$, Laffont $\mathrm{I}$ Relevance of intraglandular injections of Botulinum toxin for the treatment of sialorrhea in children with cerebral palsy: a review. Eur J Paediatr Neurol. 2014;18(6):649-657.

16. Rodwell K, Edwards P, Ware RS, Boyd R. Salivary gland botulinum toxin injections for drooling in children with cerebral palsy and neurodevelopmental disability: a systematic review. Dev Med Child Neurol. 2012;54(11):977-987.

17. Marconi B, Filippi GM, Koch G, et al. Long-term effects on cortical excitability and motor recovery induced by repeated muscle vibration in chronic stroke patients. Neurorehabil Neural Repair. 2011;25(1):48-60.
18. Tavernese $\mathrm{E}$, Paoloni $\mathrm{M}$, Mangone $\mathrm{M}$, et al. Segmental muscle vibration improves reaching movement in patients with chronic stroke. A randomized controlled trial. NeuroRehabilitation. 2013;32(3):591-599.

19. Filippi GM, Brunetti O, Botti FM, et al. Improvement of stance control and muscle performance induced by focal muscle vibration in young-elderly women: a randomized controlled trial. Arch Phys Med Rehabil. 2009:90(12):2019-2025.

20. Ribot-Ciscar E, Butler JE, Thomas CK. Facilitation of triceps brachii muscle contraction by tendon vibration after chronic cervical spinal cord injury. J Appl Physiol. 2003;94(6):2358-2367.

21. Camerota F, Galli M, Celletti C, et al. Quantitative effects of repeated muscle vibrations on gait pattern in a 5-year-old child with cerebral palsy. Case Rep Med. 2011;2011:359126.

22. Van Hulst $K$, Lindeboom R, Van der Burg J, Jongerius P. Accurate assessment of drooling severity with the 5-minute drooling quotient in children with developmental disabilities. Dev Med Child Neurol. 2012;54(12):1121-1126.

23. Reid SM, Johnson HM, Reddihough DS. The Drooling Impact Scale: A measure of the impact of drooling in children with developmental disabilities. Dev Med Child Neurol. 2010;52(2):22-28

24. Thomas-Stonell N, Greenberg J. Three treatment approaches and clinical factors in the reduction of drooling. Dysphagia. 1988;3(2):73-78.

25. Holm S. A Simple Sequentially Rejective Multiple Test Procedure. Vol 6.; 1979.

26. Erasmus CE, Van Hulst K, Rotteveel LJC, et al. Drooling in cerebral palsy: Hypersalivation or dysfunctional oral motor control? Dev Med Child Neurol. 2009;51(6):454-459.

27. Meece RW, Fishlock KF, Bayley EW, Keller MS. Ultrasound-Guided Botox Injections of Salivary Glands in Children with Drooling. J Radiol Nurs. 2010;29(1):20-24.

28. Senner JE, Logemann J, Zecker S, Gaebler-Spira D. Drooling, saliva production, and swallowing in cerebral palsy. Dev Med Child Neurol. 2004;46(12):801-806.

29. Sochaniwskyj AE, Koheil RM, Bablich K, Milner M, Kenny DJ. Oral motor functioning, frequency of swallowing and drooling in normal children and in children with cerebral palsy. Arch Phys Med Rehabil. 1986;67(12):866-874.

30. Lespargot A, Langevin MF, Muller S, Guillemont S. Swallowing disturbances associated with drooling in cerebral-palsied children. Dev Med Child Neurol. 1993;35(4):298-304.

31. Casale R, Damiani $C$, Maestri $R$, Fundarò $C$ Chimento $P$, Foti $C$. Localized 100 $\mathrm{Hz}$ vibration improves function and reduces upper limb spasticity: a double-blind controlled study. Eur J Phys Rehabil Med. 2014;50(5):495-504.

32. Calabrò RS, Naro A, Russo M, Milardi D, Leo A, Filoni S, Trinchera A, Bramanti P. Is two better than one? Muscle vibration plus robotic rehabilitation to improve upper limb spasticity and function: A pilot randomized controlled trial. PLoS One. 2017; 12(10):e0185936. 